$\begin{array}{llll}\text { Nikolaos Spilias, MD } & \text { Benjamin Zorach, MD } & \text { Kara Denby, MD } & \text { Stephen Ellis, MD } \\ \text { Fellow, Cleveland Clinic } & \text { Fellow, Cleveland Clinic } & \text { Fellow, Cleveland Clinic } & \begin{array}{l}\text { Department of Cardiovascular Medicine, Heart, } \\ \text { Vascular \& Thoracic Institute, Cleveland Clinic; } \\ \text { Professor, Cleveland Clinic Lerner College of } \\ \end{array} \\ & & \begin{array}{l}\text { Medicine of Case Western Reserve University, } \\ \text { Cleveland, OH }\end{array}\end{array}$

\title{
The role of ISCHEMIA in stable ischemic heart disease
}

\section{ABSTRACT}

Although it is well established that adding early revascularization to optimal medical therapy reduces mortality and recurrent myocardial infarction in acute coronary syndrome, there is less convincing evidence to guide intervention in stable ischemic heart disease. This review summarizes the International Study of Comparative Health Effectiveness With Medical and Invasive Approaches (ISCHEMIA) trial, which investigated whether there is benefit from initial catheterization and possible revascularization in addition to optimal medical therapy in patients with at least moderate ischemia on stress testing.

\section{KEY POINTS}

ISCHEMIA randomly assigned 5,179 patients with moderate or severe ischemia to an initial invasive strategy plus optimal medical therapy, or optimal medical therapy alone.

Over a median of 3.2 years, there was no significant difference between the 2 groups in the incidence of the primary outcome (a composite of death from cardiovascular causes, myocardial infarction, or hospitalization for unstable, angina, heart failure, or resuscitated cardiac arrest) or in a number of important secondary outcomes.

Decisions regarding treatment of stable ischemic heart disease must remain individualized.

Dr. Ellis has disclosed speaking, teaching, consulting, or serving on an advisory committee for Medtronic.

doi:10.3949/ccjm.87a.20033
Che current American College of Cardi1 ology and American Heart Association guidelines recommend coronary angiography as a "reasonable" approach (class IIA indication) in patients with suspected stable ischemic heart disease in whom the clinical characteristics and noninvasive testing indicate a high likelihood of severe coronary artery disease. ${ }^{1}$ However, uncertainty has persisted about whether to pursue an initial invasive approach as opposed to optimal medical therapy alone.

See related commentary, page 410

This review summarizes the recent International Study of Comparative Health Effectiveness With Medical and Invasive Approaches (ISCHEMIA) trial, which investigated whether there is benefit from initial catheterization and possible revascularization in addition to optimal medical therapy in patients with at least moderate ischemia on stress testing.

\section{VARIABLE FINDINGS IN EARLIER TRIALS}

Observational studies have suggested that myocardial perfusion imaging may help to riskstratify patients with stable ischemic heart disease and identify those who may benefit from revascularization. ${ }^{2,3}$ Patients with mild ischemia have been shown to have a good prognosis with optimal medical therapy alone, while those with moderate or severe ischemia seem to have survival benefit when treated with percutaneous coronary intervention (PCI) in addition to optimal medical therapy.

However, subsequent randomized controlled trials cast doubt on this notion and raised questions regarding the ideal initial management of stable ischemic heart disease. 
These trials have not shown a reduction in the rates of death or cardiovascular events with PCI compared with optimal medical therapy alone. ${ }^{4-6}$ On the other hand, PCI has been shown to reduce the rates of urgent or unplanned revascularization and spontaneous myocardial infarction, and improve angina symptoms and quality of life. - $^{4-6}$

The trials of stable ischemic heart disease treatment have been very heterogeneous in terms of design, patient selection, mode of revascularization, and medical therapies, leading to several limitations in their generalizability and applicability to current practice.

One of the main limitations was the inclusion of a broad population of patients with and without objective evidence of ischemia and without a specific threshold of required ischemia on a stress test. Patients with moderate or severe ischemia were more rarely included, based on earlier observations that the severity of ischemia may be associated with increased mortality, and revascularization may be associated with better prognosis. ${ }^{2,3}$

While the Clinical Outcomes Utilizing Revascularization and Aggressive Drug Evaluation (COURAGE) trial showed no improvement in the primary outcome of all-cause mortality and nonfatal myocardial infarction with PCI, ${ }^{4}$ a substudy showed that PCI plus optimal medical therapy was associated with a greater reduction in inducible ischemia on follow-up myocardial perfusion imaging, and those who experienced a reduction in ischemia had a lower unadjusted risk of death or myocardial infarction. ${ }^{7}$ However, in this substudy, $70 \%$ of the participants had only a small amount of ischemia $(<10 \%)$, and with risk adjustment the reduction in death and myocardial infarction was not significant. ${ }^{\text {? }}$

The COURAGE substudy, ${ }^{7}$ along with the prior observations by Hachamovitch et $\mathrm{al}^{2}{ }^{2}$ again suggested potential benefit from revascularization with more significant ischemia. $\mathrm{Nev}$ ertheless, this evidence should be used with caution, given its retrospective nature and that it comes from a substudy of a larger trial.

Further complicating the picture, in earlier trials the patient's coronary anatomy was often known before randomization, and this knowledge may have introduced bias in patient selection by limiting the inclusion of patients who had significant angiographic coronary artery disease, such as those with proximal left anterior descending or multivessel disease. ${ }^{4}$ More specifically, physicians and patients may have been reluctant to participate in a randomized trial, knowing that the coronary angiogram showed significant coronary artery disease.

Additionally, the invasive approach in previous trials was quite variable, ranging from balloon angioplasty to bare-metal stents and first-and second-generation drug-eluting stents. These trials did not use the newestgeneration drug-eluting stents, which are associated with improved outcomes. Also omitted in many studies was the use of newer invasive intravascular techniques to assess the hemodynamic significance of intermediate lesions, such as fractional flow reserve, instantaneous wave-free ratio, intravascular ultrasonography, and optical coherence tomography, which assist with the appropriate selection of lesions requiring intervention and improve revascularization outcomes. ${ }^{5}$

In the previous trials, optimal medical therapy consisted primarily of antianginal medications rather than modern diseasemodifying agents such as aspirin, statins, betablockers, and renin-angiotensin-aldosterone system inhibitors, which are now considered the foundation of medical therapy and lead to better outcomes.

Lastly, the trials were open-label, and the control groups did not undergo sham procedures, which may have introduced bias regarding the true beneficial effect of PCI in reducing angina. The Objective Randomised Blinded Investigation With Optimal Medical Therapy of Angioplasty in Stable Angina (ORBITA), ${ }^{8}$ the only randomized trial that used a sham procedure, showed no difference in exercise time in patients with stable angina undergoing PCI compared with medical therapy. This finding suggests that PCI may be associated with a placebo effect. ${ }^{8}$

Meta-analyses of stable ischemic heart disease treatment have also reported variable findings. For example, Gada et $\mathrm{al}^{9}$ performed a meta-analysis that showed a reduction in all-cause mortality with addition of PCI to optimal medical therapy. This meta-analysis included 3 randomized controlled trials 
(COURAGE Nuclear Substudy, the Fractional Flow Reserve Versus Angiography for Multivessel Evaluation 2 trial, the Swiss Interventional Study on Silent Ischemia Type II), that enrolled 1,557 patients with stable ischemic heart disease and objective evidence of myocardial ischemia by noninvasive imaging tests or fractional flow reserve. . $, 7,10$

Bangalore et $\mathrm{al}^{11}$ found a reduction in spontaneous myocardial infarctions with PCI compared with optimal medical therapy alone at the expense of periprocedural myocardial infarctions, resulting in no difference overall.

In a meta-analysis by Stergiopoulos et al, ${ }^{12}$ PCI plus optimal medical therapy was not associated with a reduction in death, myocardial infarction, unplanned revascularization, or angina compared with optimal medical therapy alone, but again, the severity of ischemia was quite variable among the different studies.

Over the past decade, PCI technology and technique have improved, and so have invasive outcomes. Similarly, improvement in primary and secondary prevention of cardiovascular disease with disease-modifying, rather than purely symptom-controlling medications, has led to equipoise and brought into question the utility of routine revascularization in stable ischemic heart disease. As a result, current practice varies widely, with many centers using stress perfusion imaging and the severity of ischemia to guide revascularization. This uncertainty set the stage for a new large randomized controlled trial in patients with stable ischemic heart disease and high-risk ischemic features.

\section{ISCHEMIA TRIAL DESIGN}

The purpose of ISCHEMIA was to evaluate if a routine initial invasive approach with cardiac catheterization and possible revascularization provides any additional benefit compared with optimal medical therapy alone in patients who have symptoms of stable ischemic heart disease and evidence of moderate or severe ischemia on stress testing.

Exclusion criteria included heart failure with reduced ejection fraction (left ventricular ejection fraction < 35\%), New York Heart Association class III or IV symptoms, hospitalization for heart failure within 6 months, coronary artery bypass grafting (CABG) or PCI within 1 year, or acute coronary syndrome within 2 months. Also excluded were patients with "severe angina despite maximal medical therapy," Canadian Cardiovascular Society class III angina of recent onset, or class IV angina, or who were "very dissatisfied" with medical management. ${ }^{13}$

From July 26, 2012, through January 31, 2018, investigators enrolled 8,518 patients and randomized 5,179 to optimal medical therapy alone vs optimal medical therapy plus an initial invasive approach, with coronary angiography followed by PCI or CABG based on decisions made by the heart team.

Most of the patients underwent blinded coronary computed tomographic (CT) angiography before randomization to exclude left main artery stenosis $(\geq 50 \%)$ and ensure the presence of significant coronary artery disease ( $\geq 50 \%$ stenosis in a major epicardial vessel for those undergoing stress imaging and $\geq 70 \%$ stenosis in a proximal or mid vessel for those undergoing exercise tolerance testing). ${ }^{13,14}$

Of the 3,339 excluded patients, $12.9 \%$ had unprotected left main disease, 36.4\% did not have obstructive coronary artery disease on CT angiography, and $40.4 \%$ did not have moderate or severe ischemia based on core laboratory assessment. ${ }^{15}$

Owing to slow enrollment, a protocol amendment in January 2014 permitted the inclusion of patients with exercise-induced ischemic electrocardiographic changes without adjunctive imaging. The inclusion criteria were also expanded to patients who demonstrated $5 \%$ or more ischemia on nuclear perfusion imaging at low levels of exertion $(\leq 7$ metabolic equivalents). ${ }^{13}$

The primary end point was originally defined in 2012 as the composite of cardiovascular death and myocardial infarction. Due to low event rates, the primary end point was expanded in 2018, just 7 months before enrollment completion, to also include resuscitated cardiac arrest, hospitalization for unstable angina, and hospitalization for heart failure. ${ }^{16}$ The myocardial infarction events included both spontaneous and periprocedural infarctions. The definitions of periprocedural myocardial infarctions (PCI- and CABG-related) included elevation in cardiac biomarkers and

\section{Over the past decade, $\mathrm{PCl}$ has improved, and so has medical therapy}


electrocardiographic changes according to the most recent proposed definition of clinically relevant myocardial infarction after revascularization, from the Society for Cardiovascular Angiography and Interventions. ${ }^{15,17}$

\section{POPULATION CHARACTERISTICS}

The median age of the study participants was 64 . Among the participants, $23 \%$ were women, and $66.3 \%$ were white. Regarding history, $73.4 \%$ had hypertension, $41.8 \%$ had diabetes, $57.4 \%$ had a history of smoking, and $19.2 \%$ had a history of myocardial infarction. Regarding angina frequency, $43.9 \%$ of the patients reported having angina monthly, $19.5 \%$ weekly, $2.3 \%$ daily, while $34.4 \%$ reported no angina in the month prior to randomization and $10.3 \%$ had no history of angina. ${ }^{14}$

Most of the patients were receiving optimal medical therapy by contemporary standards at baseline $(94.1 \%$ were receiving antiplatelet drugs, $94.8 \%$ statins at any dose, $36.7 \%$ high-intensity statins, $4.1 \%$ ezetimibe, $80.4 \%$ beta-blockers, and $66 \%$ angiotensinconverting enzyme inhibitors or angiotensin II receptor blockers). As for other antianginal
Outcomes did not differ significantly between the two treatment groups medications, long-acting nitrates were used in $32.3 \%$, calcium channel blockers in $30.5 \%$, and ranolazine in $5 \%$ of the patients. ${ }^{14}$

Regarding coronary artery disease, $75 \%$ of the patients qualified on the basis of stress imaging tests (nuclear myocardial perfusion imaging in $49 \%$, stress echocardiography in $21 \%$, cardiac magnetic resonance imaging in 5\%). According to core laboratory interpretation, $44.8 \%$ of those with stress imaging tests had severe ischemia, $41 \%$ had moderate, $8.1 \%$ had mild, and $6 \%$ had no ischemia or the test was uninterpretable..$^{14,15}$

The remaining $25 \%$ of the patients qualified on the basis of abnormal exercise tolerance testing. For these patients, stricter criteria were applied for their participation, including history of angina, an interpretable resting electrocardiogram, exercise-induced $1.5-\mathrm{mm}$ ST-segment depression in 2 leads or 2-mm STsegment depression in 1 lead or $1.5-\mathrm{mm}$ ST elevation in a noninfarct territory occurring at early stages of the exercise tolerance test, and at least $70 \%$ stenosis in a coronary artery serving a large myocardial region based on CT angiography (proximal or mid left anterior de- scending, proximal or mid right coronary artery, or proximal left circumflex artery). ${ }^{14,15}$

\section{RESULTS}

Over a median follow-up period of 3.2 years, cardiac catheterization was performed in $96 \%$ of the invasive treatment group and $28 \%$ of the optimal medical therapy group. Indications for catheterization in the optimal medical therapy group included suspected or confirmed events $(13.8 \%)$, medical therapy failure $(3.9 \%)$, and nonadherence $(8.1 \%)$. Coronary revascularization was performed in $80 \%$ of the invasive therapy group (74\% PCI, $26 \% \mathrm{CABG}$ ) and $23 \%$ of the medical therapy group. Of the $20 \%$ of the invasive therapy group who did not undergo revascularization, two-thirds had insignificant disease on angiography and the other third had extensive coronary artery disease not suitable for any mode of revascularization. ${ }^{15}$

\section{Outcomes}

Outcomes did not differ significantly between the 2 treatment groups.

The composite primary outcome (cardiovascular death, myocardial infarction, hospitalization for unstable angina, heart failure, or resuscitated cardiac arrest) occurred in 13.3\% of the invasive therapy group vs $15.5 \%$ of the optimal medical therapy group (hazard ratio [HR] 0.93, 95\% confidence interval [CI] 0.80-1.08, $P=.34$ ) (Figure 1A).

The major secondary end point (cardiovascular death or myocardial infarction occurred in $11.7 \%$ vs $13.9 \%$ ( $\mathrm{HR} 0.90,95 \% \mathrm{CI}$ $0.77-1.06, P=.21)$ (Figure 1B).

Death from any cause occurred in $6.5 \%$ vs $6.4 \%$, which were low rates $(P=.67)$ (Figure $1 \mathrm{C})$.

Myocardial infarction rates were similar (HR 0.92, 95\% CI 0.76-1.11, $P=.38$ ) (Figure 1D). However, there were more periprocedural infarctions (HR 2.98, 95\% CI 1.87-4.74, P $<.01$ ) and fewer spontaneous infarctions (HR $0.67,95 \%$ CI $0.53-0.83, P<.01)$ in the invasive therapy group.

Hospitalizations. The invasive therapy group had fewer hospitalizations for unstable angina (HR 0.50, 95\% CI 0.27-0.91, $P=.02$ ), but more hospitalizations for heart failure (HR 2.23, 95\% CI 1.38-3.61, $P<.01)$. 


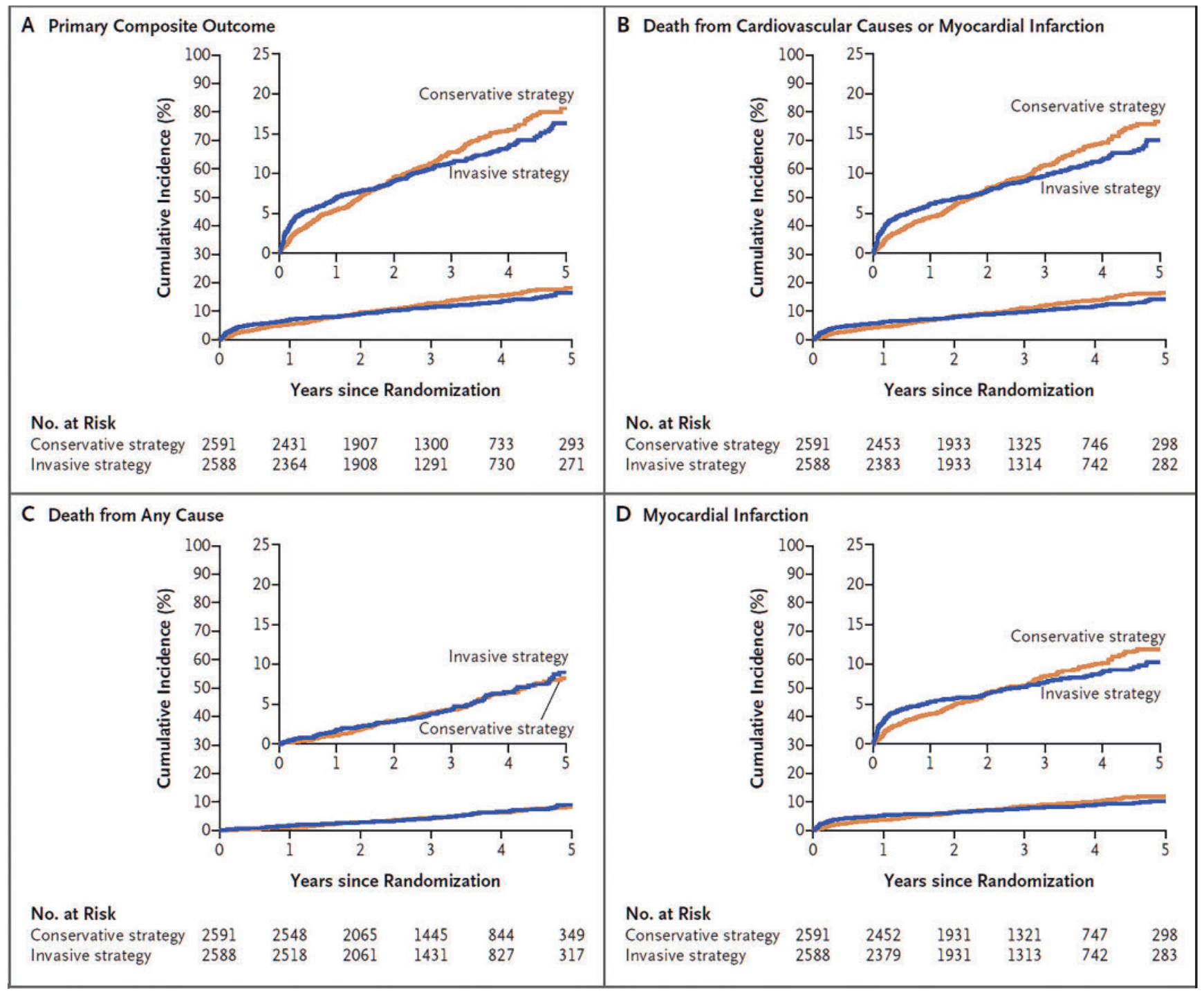

Figure 1. Time-to-event curves in the ISCHEMIA trial. The primary composite outcome consisted of death from cardiovascular causes, myocardial infarction, or hospitalization for unstable angina, heart failure, or resuscitated cardiac arrest.

From Maron DJ, Hochman JS, Reynolds HR, et al. Initial invasive or conservative strategy for stable coronary disease. N Engl J Med 2020; 382(15):1395-1407. Copyright 2020, Massachusetts Medical Society. Reprinted with permission of the Massachusetts Medical Society.

Stroke and resuscitated cardiac arrest rates were similar between the 2 groups. ${ }^{15}$

Angina. The invasive therapy group experienced more reduction in angina frequency at 3 months than the optimal medical therapy group. In terms of quality of life, patients with moderate or severe ischemia and frequent angina (daily, weekly, or monthly) had better angina control with the invasive strategy. ${ }^{15}$

The outcomes were similar between the 2 groups irrespective of the type of stress modality used, severity of ischemia, or extent of coronary artery disease on CT angiography. ${ }^{15}$

\section{STRENGTHS OF THE TRIAL}

ISCHEMIA was the first large randomized controlled trial in the field of stable ischemic heart disease to include mainly patients with moderate to severe ischemia on stress testing as well as anatomic evidence of coronary ar- 
tery disease based on CT angiography. Various forms of stress tests were used to quantify ischemia, including nuclear myocardial perfusion imaging, stress echocardiography, stress magnetic resonance imaging, and exercise electrocardiography, with the inclusion criteria for the latter being stricter, as described above.

Unlike previous trials, ISCHEMIA did not require the coronary anatomy to be angiographically defined before randomization, thus reducing possible selection bias. Moreover, up-front knowledge of coronary anatomy could increase the risk of ascertainment bias among providers and patients by potentially increasing reported events and crossovers in the optimal medical therapy group.

Although the coronary anatomy was not fully defined, most of the patients were screened with CT angiography before randomization to exclude significant left main artery disease and to ensure the presence of coronary artery disease in an effort to minimize the inclusion of patients with false-positive stress tests. Based on CT angiography, most patients had evidence of highrisk coronary artery disease, as reflected in the disease of multiple vessels in $79 \%$, left anterior descending artery in $86.8 \%$, and proximal left anterior descending artery in $46.8 \%{ }^{14,15}$

One of the main strengths of ISCHEMIA was the use of contemporary revascularization strategies: $98 \%$ of the patients in the PCI group received latest-generation drug-eluting stents, and $93 \%$ in the CABG group received arterial grafts. Additional evaluation of intermediate lesions was performed with the use of the most advanced available technology in the catheterization laboratory (fractional flow reserve, instantaneous wave-free ratio, intravascular ultrasonography), although their use was relatively limited. ${ }^{18}$

\section{LIMITATIONS}

\section{OF THE TRIAL}

Although ISCHEMIA was originally designed to include only patients with moderate or severe ischemia on stress imaging, challenges with recruitment led to the inclusion of patients with less ischemia as well as patients who met only the exercise tolerance testing criteria. ${ }^{13}$ In fact, $14.1 \%$ of patients who underwent stress imaging and $9 \%$ of those who underwent exercise tolerance testing had mild or no ischemia or an uninterpretable stress test based on core laboratory assessment. ${ }^{14}$ This was addressed by the authors, who found no effect on the primary outcome in an analysis of heterogeneity of treatment effect. ${ }^{15}$ Exercise electrocardiographic testing without imaging was also used more often (in 24.5\% ) $^{14}$ than in contemporary practice, in which imaging modalities are generally preferred for the assessment and quantification of ischemia.

Perhaps also related to poor enrollment was the inclusion of patients with no angina (10.3\%) and patients who had not had angina within the month before randomization $(34.4 \%) .{ }^{14}$ While this certainly represents a subset of patients who undergo stress testing (eg, during preoperative assessment in patients who cannot accomplish 4 metabolic equivalents), it is unclear whether those without symptoms or those whose symptoms have subsided are at the same risk as those with active or more significant burden of angina. More importantly, if this subset of patients with minimal symptoms overlapped considerably with those with mild or no ischemia, they may represent a low-risk population and their inclusion may have attenuated the potential benefit of an invasive strategy.

A second limitation of the study was the large proportion of outcome events that were myocardial infarctions, either periprocedural or spontaneous. This in part was due to low mortality rates relative to the rates of myocardial infarction and the other measures included in the composite primary outcome. Early on, rates of periprocedural myocardial infarction were higher in the invasive treatment group, but later, rates of spontaneous myocardial infarction were higher in the optimal medical therapy group. While these rates are combined in the outcome of total myocardial infarctions, the authors state that a preliminary analysis of ISCHEMIA data supports the findings of previous studies showing that spontaneous myocardial infarction is associated with higher morbidity and mortality rates than periprocedural myocardial infarction. ${ }^{15,19}$ Furthermore, given the trends noted in the time-to-event curves, the 2 groups may continue to diverge in the primary composite outcome, with lower rates in the invasive 
therapy group. Therefore, longer follow-up is warranted to fully understand the prognostic implications of the different spontaneous and periprocedural myocardial infarction rates between the 2 groups. ${ }^{20}$

A third and probably the most important limitation is the applicability of ISCHEMIA results to current practice. Changing the primary end point to include "softer" and more subjective clinical end points such as hospitalization for heart failure or unstable angina, as well as including patients with less ischemic burden than originally planned, raises concerns about the trial's applicability to clinical practice and ability to answer the main study question.

Additionally, only $22.6 \%$ of the study participants were women, and women were more often excluded for having less ischemia on stress testing and less obstructive coronary artery disease on CT angiography. ${ }^{14}$

Importantly, patient groups who were excluded, such as those with heart failure, significant angina, or revascularization within a year, represent a significant portion of patients with stable ischemic heart disease symptoms encountered in daily clinical practice.

Several features of the ISCHEMIA trial were not completely addressed in its publications or supplementary materials. For example, more information is needed about the use of intravascular ultrasonography or physiologic measures such as instantaneous wave-free ratio or fractional flow reserve in guiding coronary interventions in the invasive group. The appendix reports only generally on fractional flow reserve, stating that it was used in $20.3 \%$ of patients in the invasive therapy group in their initial catheterization, but does not detail how that influenced treatment decisions. ${ }^{18}$ Although these strategies are not routinely used in most PCI cases, the benefit from their use in reduction of major adverse cardiovascular events and improvement in interventional outcomes has been well established. ${ }^{21,22}$

Finally, a comparison between the PCI and $C A B G$ subgroups of the invasive therapy group in terms of patient characteristics, severity and location of coronary lesions, completeness of revascularization, and outcomes is not included in the publication.

\section{CLINICAL IMPLICATIONS}

Based on the findings of the trial, the utility of stress testing in the assessment of stable ischemic heart disease is brought into question, as the presence of moderate or severe ischemia did not seem to lead to severe adverse outcomes regardless of invasive or conservative approach. Additionally, the trial shows the weaknesses of these assessments as tools to reliably diagnose obstructive coronary disease; a modest proportion (21\%) of patients did not have $50 \%$ or greater stenosis on CT angiography, showing the differences between anatomic evidence of epicardial coronary artery disease and physiologic evidence of ischemia. ${ }^{14}$ This finding could also be related to the inclusion of patients with mild or no ischemia as described above. Furthermore, about $15 \%$ of patients in the invasive group did not have obstructive coronary artery disease on angiography, highlighting the significant rate of false-positive stress tests. ${ }^{15}$

As noted, an important group that was excluded was patients with left main stenosis of $50 \%$ or more on CT angiography ( $7.5 \%$ of the patients who underwent this test). ${ }^{14}$ By virtue of this protocol, an anatomic study (CT angiography or cardiac catheterization) would be necessary for a patient undergoing evaluation for stable angina in order to exclude left main disease. This may lead to more providers obtaining anatomic studies initially, potentially at the expense of stress testing, in the evaluation of patients with stable angina. While outside the scope of this review, available data about the use of CT angiography in suspected stable ischemic heart disease have not shown improvement in "hard" clinical outcomes compared with functional stress testing, although it led to fewer "unnecessary" catheterizations showing no obstructive coronary artery disease. ${ }^{23}$

Therefore, the optimal sequence of diagnosing obstructive coronary artery disease and evaluating stable coronary disease is in question. Given the false-positive rate of functional stress tests and need to exclude left main stenosis, up-front evaluation with CT angiography may be warranted in many cases.

When significant left main disease has been excluded, the provider should addition-
The findings of the trial have brought into question the utility of stress testing in assessment of stable ischemic heart disease 
ally ensure that the patient is similar to the population enrolled in ISCHEMIA, taking into account the other exclusion criteria. If that is the case, proceeding with either an initial invasive strategy or conservative approach will then require an informed decision that will vary based on individual patient factors after risk/benefit discussion.

\section{CONCLUSIONS AND FUTURE DIRECTIONS}

The results of ISCHEMIA are consistent with those of previous trials in patients with stable ischemic heart disease, suggesting that despite a reduction in symptoms, angina-related hospitalizations, and spontaneous myocardial infarction, there is no clear survival benefit from an initial invasive strategy compared with optimal medical therapy alone after a follow-up period of 3.2 years, even in patients with moderate or severe ischemia.

Taking into account the limitations de-

\section{REFERENCES}

1. Patel MR, Calhoon JH, Dehmer GJ, et al. ACC/AATS/ AHA/ASE/ASNC/SCAI/SCCT/STS 2017 appropriate use criteria for coronary revascularization in patients with stable ischemic heart disease. J Am Coll Cardiol 2017; 69(17):2212-2241. doi:10.1016/j.jacc.2017.02.001

2. Hachamovitch R, Hayes SW, Friedman JD, et al. Comparison of the short-term survival benefit associated with revascularization compared with medical therapy in patients with no prior coronary artery disease undergoing stress myocardial perfusion single photon emission computed tomography. Circulation 2003; 107(23):2900 2907. doi:10.1161/01.CIR.0000072790.23090.41

3. Hachamovitch R, Rozanski A, Shaw LJ, et al. Impact of ischaemia and scar on the therapeutic benefit derived from myocardial revascularization vs. medical therapy among patients undergoing stress-rest myocardial perfusion scintigraphy. Eur Heart J 2011; 32(8):1012-1024. doi:10.1093/eurheartj/ehq500

4. Boden WE, O'Rourke RA, Teo KK, et al; COURAGE Trial Research Group. Optimal medical therapy with or with out PCl for stable coronary disease. N Engl J Med 2007; 356(15):1503-1516. doi:10.1056/NEJMoa070829

5. De Bruyne B, Pijls NH, Kalesan B, et al; FAME 2 Trial Investigators. Fractional flow reserve-guided $\mathrm{PCl}$ versus medical therapy in stable coronary disease. N Engl J Med 2012; 367(11):991-1001. doi:10.1056/NEJMoa1205361

6. Frye RL, August P, Brooks MM, et al; BARI 2D Study Group. A randomized trial of therapies for type 2 diabetes and coronary artery disease. N Engl J Med 2009; 360(24):2503-2515. doi:10.1056/NEJMoa0805796

7. Shaw LJ, Berman D, Maron D, et al; COURAGE Investigators. Optimal medical therapy with or without percutaneous coronary intervention to reduce ischemic burden: results from the Clinical Outcomes Utilizing Revascularization and Aggressive Drug Evaluation (COURAGE) trial nuclear substudy. Circulation 2008; 117(10):1283-1291. doi:10.1161/CIRCULATIONAHA.107.743963 scribed above, it is not apparent that the results of this trial will significantly alter the current practice of stable ischemic heart disease management. Deciding between an initial invasive vs a conservative approach in patients who present with stable angina has beenand should continue to be-individualized, based on patient preference, angina severity, ability to tolerate optimal doses of antianginal therapy, availability of diagnostic testing, and risk of procedural complications associated with coronary interventions.

Future studies will need to address the optimal sequence and selection of noninvasive testing to better risk-stratify patients presenting with symptoms of stable ischemic heart disease. Identifying patients who may benefit from an initial invasive approach as well as the optimal management of the patient groups not included in ISCHEMIA should be the focus of future randomized trials.

8. Al-Lamee $R$, Thompson D, Dehbi HM, et al. ORBITA investigators. Percutaneous coronary intervention in stable angina (ORBITA): a double-blind, randomised controlled trial. Lancet 2018; 391(10115):31-40. doi:10.1016/S0140-6736(17)32714-9

9. Gada H, Kirtane AJ, Kereiakes DJ, et al. Meta-analysis of trials on mortality after percutaneous coronary intervention compared with medical therapy in patients with stable coronary heart disease and objective evidence of myocardial ischemia. Am J Cardiol 2015; 115(9):11941199. doi:10.1016/j.amjcard.2015.01.556

10. Erne P, Schoenenberger AW, Burckhardt D, et al. Effects of percutaneous coronary interventions in silent ischemia after myocardial infarction: the SWISSI II randomized controlled trial. JAMA 2007; 297(18):1985-1991. doi:10.1001/jama.297.18.1985

11. Bangalore S, Pursnani S, Kumar S, Bagos PG. Percutaneous coronary intervention versus optimal medical therapy for prevention of spontaneous myocardial infarction in subjects with stable ischemic heart disease. Circulation 2013; 127(7):769-781. doi:10.1161/CIRCULATIONAHA.112.131961

12. Stergiopoulos $K$, Boden WE, Hartigan $P$, et al. Percutaneous coronary intervention outcomes in patients with stable obstructive coronary artery disease and myocardial ischemia. A collaborative meta-analysis of contemporary randomized clinical trials. JAMA Intern Med 2014; 174(2):232-240. doi:10.1001/jamainternmed.2013.12855

13. ISCHEMIA Trial Research Group, Maron DJ, Hochman JS, O'Brien SM, et al. International Study of Comparative Health Effectiveness with Medical and Invasive Approaches (ISCHEMIA) trial: rationale and design. Am Heart J 2018 Jul; 201:124-135. doi:10.1016/j.ahj.2018.04.011

14. Hochman JS, Reynolds HR, Bangalore S, et al; ISCHEMIA Research Group. Baseline characteristics and risk profiles of participants in the ISCHEMIA randomized clinical trial. JAMA Cardiol 2019; 4(3):273-286. doi:10.1001/jamacardio.2019.0014 
15. Maron DJ, Hochman JS, Reynolds HR, et al. Initial invasive or conservative strategy for stable coronary disease. N Engl J Med 2020; 382(15):1395-1407. doi:10.1056/NEJMoa1915922

16. Bangalore S, Maron DJ, Reynolds HR, et al. ISCHEMIA: establishing the primary endpoint. Circ Cardiovasc Qual Outcomes 2018; 11(5): e004791. doi:10.1161/CIRCOUTCOMES.118.004791

17. Moussa ID, Klein LW, Shah B, et al. Consideration of a new definition of clinically relevant myocardial infarction after coronary revascularization: an expert consensus document from the Society for Cardiovascular Angiography and Interventions (SCAI). Catheter Cardiovasc Interv 2014; 83(1):27-36. doi:10.1002/ccd.25135

18. Maron DJ, Hochman JS, Reynolds HR, et al. Supplement to: Initial invasive or conservative strategy for stable coronary disease. N Engl J Med 2020; 382(15):1395-1407. doi:10.1056/NEJMoa1915922

19. Prasad A, Gersh BJ, Bertrand ME, et al. Prognostic significance of periprocedural versus spontaneously occurring myocardial infarction after percutaneous coronary intervention in patients with acute coronary syndromes: an analysis from the ACUITY (Acute Catheterization and
Urgent Intervention Triage Strategy) trial. J Am Coll Cardiol 2009; 54(5):477-486. doi:10.1016/j.jacc.2009.03.063

20. Antman EM, Braunwald E. Managing stable ischemic heart disease. N Engl J Med 2020; 382(15):1468-1470. doi:10.1056/NEJMe2000239

21. Zhang $D$, Lv S, Song $X$, et al. Fractional flow reserve versus angiography for guiding percutaneous coronary intervention: a meta-analysis. Heart 2015; 101(6):455462. doi:10.1136/heartjnl-2014-306578

22. Elgendy IY, Mahmoud AN, Elgendy AY, Bavry AA. Outcomes with intravascular ultrasound-guided stent implantation: a meta-analysis of randomized trials in the era of drug-eluting stents. Circ Cardiovasc Interv 2016; 9(4): 003700 doi:10.1161/CIRCINTERVENTIONS. 116.003700

23. Douglas PS, Hoffmann U, Patel MR, et al; PROMISE Investigators. Outcomes of anatomical versus functional testing for coronary artery disease. N Engl J Med 2015; 372(14):1291-1300. doi:10.1056/NEJMoa1415516

Address: Stephen Ellis, MD, Department of Cardiovascular Medicine, J2-3, Cleveland Clinic, 9500 Euclid Avenue, Cleveland, $\mathrm{OH}$ 44195; elliss@ccf.org 